\title{
Theoretical Analysis on the Possible Interruption of Cell Division by Applying Physical Excitation to Gold Nanoparticles Introduced in Chromosomes
}

\author{
Yujiro Naruse
}

Japan Science and Technology Agency, Japan

\begin{abstract}
Theoretical analysis on cell division control using physical excitations for biocompatible gold nanoparticles in chromosomes is performed. The methods of physical excitations are utilizing ultrasound, electromagnetic waves and $X-$ rays. Due to the mass increase of chromosomes, ultrasound enhances the vibration of chromosomes in metaphase of mitosis, which will disturb the alignment of chromosomes on metaphase plates and will suppress cell divisions. Under the exposure of electromagnetic waves, gold wires composed of gold nanoparticles will act as antennas in chromosomes, which will absorb electromagnetic energy and increase the temperature of chromosomes. There is a possibility that the temperature rise will bring about the transformation of DNA structure and interrupt the mitosis process. Gold nanoparticles exposed by X-rays in chromosomes emit high energy electrons through photoelectric effect, which will bring about a possibility that the high energy electrons will break the DNA structure and suppress mitosis process.
\end{abstract}

Keywords: Gold nanoparticles, cell membrane, chromosome, histone, ultrasound, electromagnetic wave, X-ray.

\section{INTRODUCTION}

Recently nanotechnology is widely recognized as leading innovations in various fields, such as integrated circuits, material science and medical science. For example, AFM (Atomic Force Microscope) and DDS (Drug Delivery System) are promising in the medical diagnostics and medical treatments, respectively. In future there will be many innovations in nanobiotechnology.

This paper proposes the novel methods to control the growth of target cells, which will lead to new cancer treatments.

The basic principle is explained in the following using the cell division cycle depicted in Figure 1a, where the definition of the stages are as follows.

stage-1: gold nanoparticles are approaching to the target cells

stage-2: gold nanoparticles are close to cell membranes

stage-3: gold nanoparticles are around condensed chromosomes in metaphase of mitosis

stage-4: gold particles are confined in nucleuses

*Address correspondence to this author at the Japan Science and Technology Agency, Japan; Tel: +81-3-3512-3523; Fax: +81-3-3222-2069;

E-mail: y2naruse@jst.go.jp
In the stage 1, the gold nanoparticles are moving towards the targeted zone such as cancer cells. One of the useful mechanisms is EPR (enhanced permeability and retention) effect which will occur in the blood vessels around the cancer cellular tissues [1]. The other method is controlled diffusion using graded electric fields of electromagnetic waves [2]. Using this method, there are possibilities for controlling the movement of gold particles and also electroporation by electromagnetic waves.

In the stage 2, the gold nanoparticles are gathering around the cell membranes. In order to get into the cytoplasm systematically through cell membranes, it is necessary for gold nanoparticles to obtain energy from physical fields. Examples of gaining energy are electroporation by gold nanoparticles excited by electromagnetic waves [2] and mechanical vibration [3]. However there is an evidence for gold particles to get into cytoplasm by surface functionalization of gold nanoparticles without physical excitation [4].

In stage 3, gold particles are able to approach to condensed chromosomes in metaphase of cell division, as the nucleus membrane is vanished in this phase.

After the metaphase of cell cycle, the cell is divided into two cells shown in stage 4 . In each cell, the nucleus membranes are formed again, and some of the gold nanoparticles will be absorbed into the nucleus.

Before the next cell cycle resumes, the gold nanoparticles are mixed and floating with uncondensed DNA fibers shown in stage-4 of Figure 1a. However the 


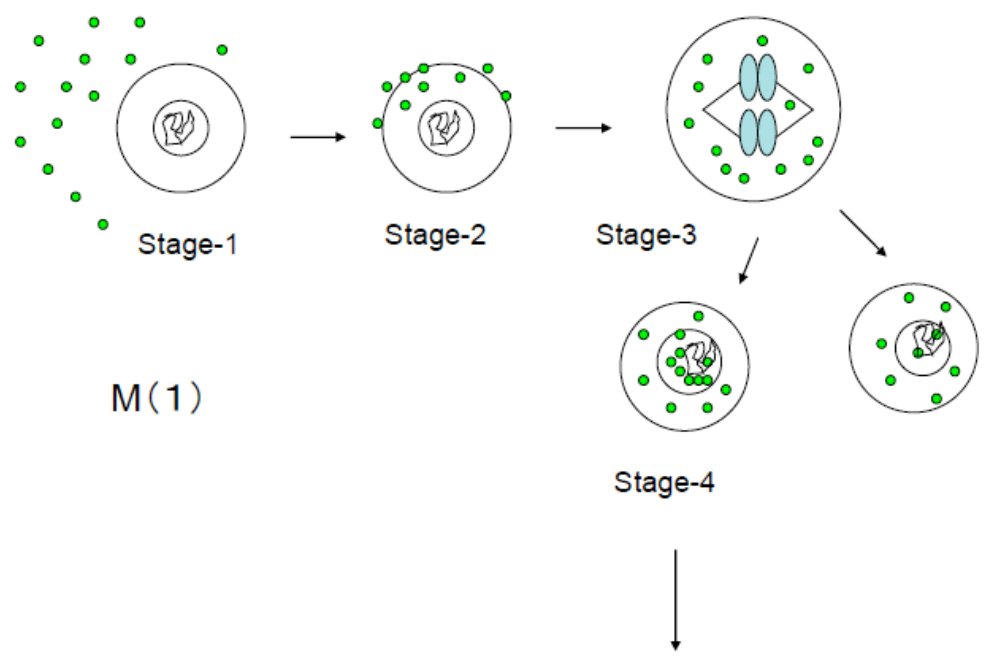

$\mathrm{M}(2 \sim \mathrm{n})$

(a)

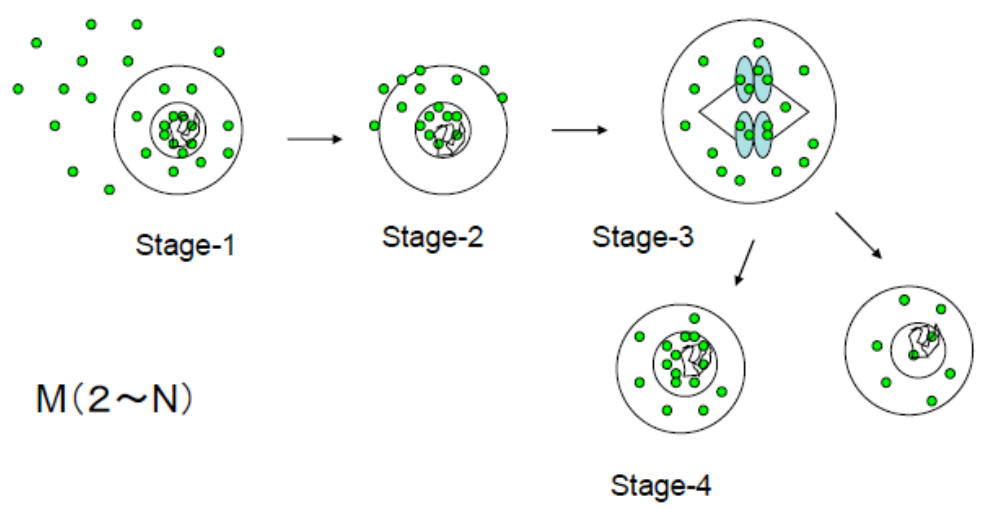

(b)

Figure 1: The schematic representation for introduction of gold nanoparticles into chromosomes.

(a) First cell cycle: $M(1)$.

(b) From the second cell cycle: $M(2 \sim n)$.

DNA fiber will be gradually condensed around histones and gold particles towards the starting of next cell division, as is shown in Figure $\mathbf{1 b}$.

In the second cell cycle shown in Figure $\mathbf{1 b}$, the gold naoparticles already exist in the cytoplasm and cell nucleus. So that, in the process of forming condensed chromosomes, gold nanoparticles will be also coiled into chromosomes like histones.

In order to enhance the probability for gold particles being coiled by DNA, it is recommended that the surfaces of the gold particles are modified by positively charged materials. This is because the DNA is negatively charged in cells.

In accordance with cell cycles $(\mathrm{M}=2 \sim \mathrm{N}$ in Figure 1b), the density of gold particles in chromosomes will increase. Especially in the case of cancer cells, gold nanoparticles will be introduced into their chromosomes rapidly because of high frequency of cell divisions.

And once the number of gold nanoparticles introduced into the condensed chromosomes reach to some level, the cell division control is possible by the following three kinds of physical excitations, which will be discussed in the next section.

\section{THEORETICAL PREDICTION FOR THE EFFECT ON CELL DIVISION BY PHYSICAL EXCITATION OF GOLD NANOPARTICLES IN CHROMOSOMES}

In this section, three kinds of physical excitation are considered in terms of cell division control. They are excitation by ultrasound, electromagnetic wave and X-ray. 


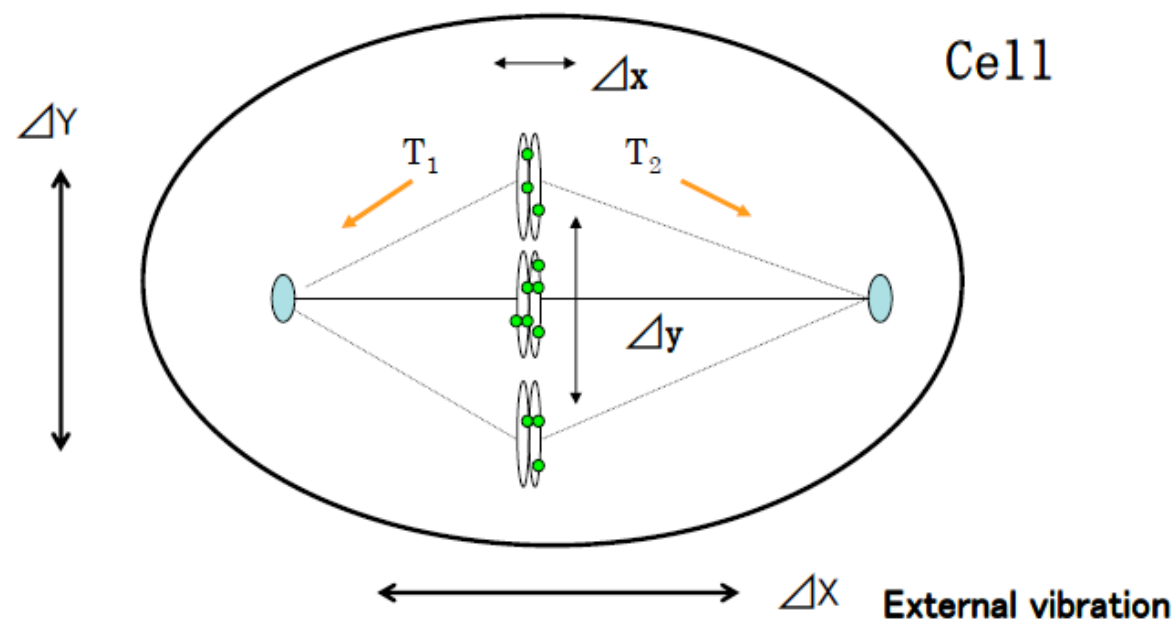

Figure 2: Schematic representation of mechanical vibration model for chromosomes in metaphase of mitosis.

\subsection{Excitation by Ultrasound}

The basic equation for the $\mathrm{H}$-mode vibration (parallel to metaphase plate) of chromosomes in metaphase of mitosis is given as follows [5].

$$
\mathrm{M} \triangle \mathrm{y} "+\mathrm{C} \Delta \mathrm{y}^{\prime}+\mathrm{k} \Delta \mathrm{y}=\mathrm{P} \cos (\omega \mathrm{t})
$$

Where, y" and y' are defined as first and second time differentials, respectively, and $\mathrm{M}, \mathrm{C}, \mathrm{k}$ and Pcos (wt) are mass of the chromosome, viscous damping constant, spring constant and external vibration caused by ultrasound, respectively.

From equation (1), the maximum amplitude for is calculated as follows.

$A \max =2 M P / C\left(4 M k+C^{2}\right)^{0.5}$

As the Amax given by the equation (2) is a monotonously increasing function of the mass of $M$, it is easily understood that Amax will increase in proportion to the number of gold nanoparticles introduced in chromosomes. In addition mass of the gold nanoparticle with diameter $10 \mathrm{~nm}$ is much larger than the histones shown in Table 1.

Consequently gold nanoparticles in chromosomes under ultrasound vibration will suppress the cell division through the disturbance of the alignment of chromosomes on the metaphase plates [5].

\subsection{Excitation by Electromagnetic Waves}

In the condensation process of chromosomes, the distances between gold nanoparticles will be shortened, and eventually virtual gold wires will emerge shown in Figure 3.

Under the exposure of electromagnetic waves the gold wires play the role of antenna and the induced voltage between $a$ and $b$ in Figure $\mathbf{3}$ is expressed as follows.

$\mathrm{V}=\int_{\mathrm{a}}^{\mathrm{b}} \mathbf{E} \bullet \boldsymbol{d l}$

where $\mathrm{E}$ is incident electric field vector, $\cdot \mathrm{dl}$ is the elemental operator of inner product for integrating along the line of the gold wire between the edges of the points $a$ and $b$, shown in Figure 3.

For a simplified ideal case, the temperature increase per second $\Delta \mathrm{T}$ can be written as the following equation which is not dependent on the shape of the gold wire.

$\Delta T=E^{2} /(\rho \cdot h \cdot c)$

where $\rho$ is resistivity, $h$ is specific density and $c$ is specific heat for gold.

Table 1: Comparison of Masses Between Gold Nanoparticle and Histones [kDa]

\begin{tabular}{|c|c|c|c|c|}
\hline Au particle & H1 & H2A & H2B & H3 \\
\hline \hline 6086 & 23 & 14 & 13.8 & 15.3 \\
\hline
\end{tabular}

Note: Au particle with diameter $10 \mathrm{~nm}$ is equivalently calculated as $6086 \mathrm{kDa}$.

Data of histones are cited fromp521 of reference [6] 


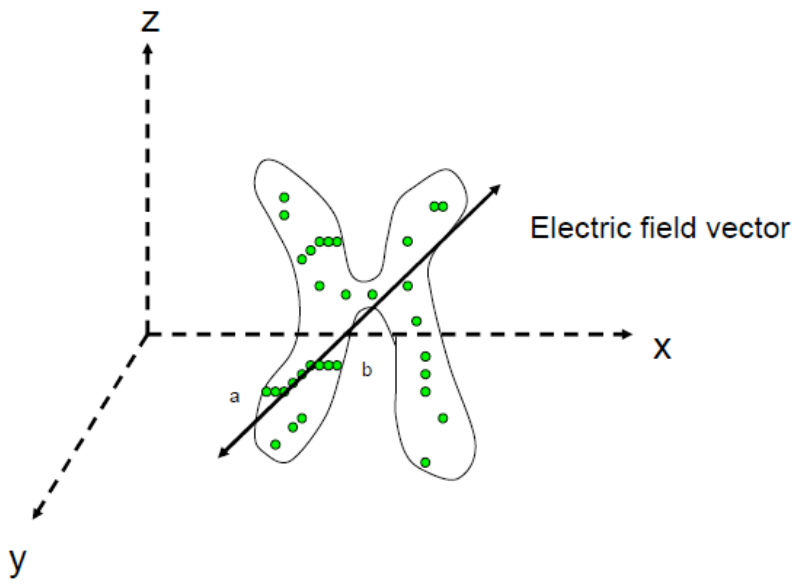

Figure 3: Schematic representation of gold wires composed of gold nanoparticles under the exposure of electric field.

Equation (4) is derived based on the following assumptions.

>> the gold wire is straight and in parallel with the electric field vector of the electromagnetic wave.

>> two edges of the gold wire are earthed to electrolyte (cytoplasm): there is no charge storage on gold wires.

In order to estimate an example of the temperature increase per second of a gold wire, the following parameters are assumed.

>> Effective strength of electric field $E$ is $1 \mathrm{~V} / \mathrm{m}$

$>\rho: 2.4\left[10^{-8} \Omega \cdot m\right]$ $>>c: 130[\mathrm{~J} /(\mathrm{Kg} \cdot \mathrm{K})]$

$>\mathrm{h}: 1.93\left[10^{4} \mathrm{Kg} / \mathrm{m}^{3}\right]$

onsequently $\Delta \mathbf{T}$ is approximately calculated as 16.6 $\left[{ }^{\circ} \mathrm{C} / \mathrm{sec}\right]$.

\subsection{Excitation by X-Rays}

Hisones are mainly composed of low- $Z$ (atomic number) elements, such as $\mathrm{C}, \mathrm{O}, \mathrm{N}$ and $\mathrm{H}$. Table 2 show the mass absorption coefficient for gold and the elements in histones at the X-ray photon energy of $50 \mathrm{KeV}$ which is around the average energy for X-ray diagnostic systems. From the table 2 it is obvious that $X$-ray will interact far more strongly with gold nanoparticles than histones. Furthermore, the interaction between $\mathrm{X}$-ray and high-Z gold is mainly photoelectric effect which produces high energy electrons. These high energy negatively charged electrons strongly interact with surrounding DNA and bring about disturbance for mitosis process through the deterioration of DNA.

The utilization of high energy electrons emitted from high-Z materials is similar to the high sensitivity X-ray detector having periodical multilayer structure with high and low-Z elements [7].

\section{SUMMARY}

Theoretical analysis on the behavior of gold nanoparticles introduced in chromosomes under

Table 2: Mass Absorption Coefficient for Elements at $50 \mathrm{KeV}\left[\mathrm{cm}^{2} / \mathrm{g}\right]$

\begin{tabular}{|c|c|c|c|c|}
\hline Au & C & O & N & H \\
\hline \hline 7.256 & 0.187 & 0.213 & 0.198 & 0.336 \\
\hline
\end{tabular}

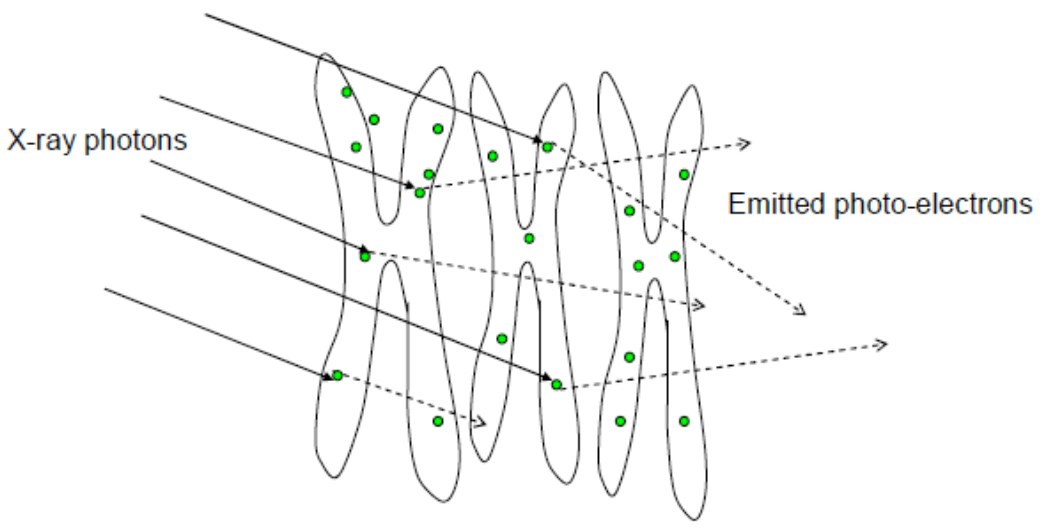

Figure 4: Schematic representation for interaction between X-ray photons and gold nanoparticles introduced in chromosomes. 
physical fields (ultrasound, electromagnetic waves and X-ray) revealed that there are three kinds of possible methods for suppressing mitosis process.

Firstly, due to the mass increase of chromosomes, ultrasound enhances the vibration of chromosomes in metaphase of mitosis, which will disturb the alignment of chromosomes on metaphase plates and will suppress cell divisions.

Secondly, gold wires composed of gold particles will act as antennas under the exposure of electromagnetic waves, which absorb electromagnetic energy and increase the temperature of chromosomes. There is a possibility that the temperature rise will bring about the transformation of DNA structure and suppress the mitosis process.

Thirdly, X-rays interact with gold nanoparticles in chromosomes and emit high energy electrons through photoelectric effect. There is a possibility that the interaction between high energy electrons and DNA will break the DNA structure and suppress mitosis process.

In order to make the concepts proposed in this paper approach real applications, further studies as for the following items are needed.

1. Development of efficient transportation of gold nanoparticles into chromosomes.

2. Computer simulation for dynamic behavior of gold nanoparticles introduced in chromosomes under ultrasound, electromagnetic waves and Xrays.

3. Experiments using living cells and gold nanoparticles.

\section{REFERENCES}

[1] Maeda H. Tumor-selective delivery of macromolecular drugs via EPR effect: Background and future prospects. Bioconjug Chem 2010; 21: 797.

http://dx.doi.org/10.1021/bc100070g

[2] Naruse Y. Diffusion control of strongly correlated electrically conductive particles in liquid medium by applying graded electromagnetic fields, IEEJ transactions on electrical and electronic engineering, IEEJ Trans 2012, published online in Wiley Online Library (wileyonlinelibrary.com).

[3] Naruse Y. Mechanical vibration model for proteins and nanoparticles coupling with the cell membrane and its application to cell treatment. Japanese J Appl Phys 2004; 43 : 3629.

http://dx.doi.org/10.1143/JJAP.43.3629

[4] Shenoy D, et al. Surface functionalization of gold nanoparticles using hetero-bifunctional poly(ethylene glycol)spacer for intracellular tracking and delivery. Int $J$ Nanomed 2006; 1(1): 51-57. http://dx.doi.org/10.2147/nano.2006.1.1.51

[5] Naruse Y. Mechanical vibration model for chromosomes in metaphase of mitosis and possible application to the interruption of cell division. BioSystems 2002; 66: 55-63. http://dx.doi.org/10.1016/S0303-2647(02)00033-3

[6] Karp GC. Cell and Molecular Biology (Concepts and Experiments), 4th ed. John Wiley \& Sons Inc, New York, USA 2005.

[7] Naruse Y. Metal/amorphous silicon multilayer radiation detectors. IEEE Trans Nuclear Sci 1989; 36(2): 1347-52. http://dx.doi.org/10.1109/23.25528 